\title{
IN MEMORIAM JOHANNES OFFERHAUS
}

par

\section{I. DE WINTER}

Le 22 septembre, à l'âge de 74 ans, est décédé le Professeur Offerhaus au cours d'un séjour de vacances en Italie. Aucun de ceux qui l'avaient rencontré peu avant son départ n'aurait pu se douter de cette fin. Lorsqu'en avril dernier, à l'occasion de l'ouverture de la Session extraordinaire, La Conférence de La Haye de Droit International Privé lui a exprimé sa reconnaissance et lui a rendu hommage pour ses grands mérites à l'égard de cette organisation internationale, Offerhaus était l'homme qu'il avait toujours été: élancé, la figure jeune, l'air quelque peu autoritaire, écoutant avec attention et en apparence sans émotion les paroles élogieuses qui lui étaient adressées.

Son intellect, son esprit non plus n'avaient pas changé. Même pendant ses dernières vacances il se vouait encore, avec zèle et enthousiasme, à la préparation d'un rapport sur le fait illicite pour l'Institut de Droit International et quelques jours seulement avant son départ pour l'Italie il a envoyéà la rédaction de cette Revue une nécrologie sur Hessel Yntema qui - triste coïncidence - paraît dans ce même numéro où nous lui rendons hommage.

En 1962, lorsqu'il s'est retiré du professorat, cette Revue a publié un numéro spécial, portant le titre "De Conflictu Legum ", comme hommage à Offerhaus et à son collègue de Leyde, $M$. Kollewijn, qui tous deux atteignaient l'âge de yo ans cette année-là. Ge volume contient une biographie d'Offerhaus de la main du Professeur Kisch. Il donne une esquisse magistrale de la vie d'Offerhaus: brillant étudiant, recteur de l'Association des étudiants d'Amsterdam, thèse sur le droit international de la preuve, qui lui valait la plus haute distinction néerlandaise, la mention "cum laude ", avocat estimé à Rotterdam où il devint une autorité en matière de droit maritime, professeur de droit commercial et de droit international privé à l'Université d'Amsterdam, Président de la Nederlandsche Vereniging voor Zeerecht, Président du Raad voor de Scheepvaart, Président de la Commission d'Etat néerlandaise pour la codification du droit international privé et, depuis I95 I, Président de toutes les Sessions de La Conférence de La Haye.

Mais le fait d'avoir atteint l'âge de 70 ans n'a nullement mis fin à la remarquable carrière d'Offerhaus. En 1964 il a encore présidé la Dixième Session de La Conférence de La Haye et en cette même année il a été président de la Nederlandse Juristenvereniging; jusqu'en 1965 il a fait fonction de Président de la Commission 
d'Etat pour la codification du droit international privé. En 1962 l'Université Libre de Bruxelles lui conféra le doctorat honoris causa, en I 963 l'Université de Berne en fit de même. Lors de sa démission comme Président de la Commission d'Etat Sa Majesté la Reine a daigné le nommer Grand Officier de l'Ordre d'Orange-Nassau, distinction qui lui a été remise par le Ministre de la Justice.

Peu d'hommes ont tant accompli dans leur carrière, à peu d'hommes tant d'honneurs sont échus.

Pour ses amis et pour tous ceux qui ont eu le privilège de le connaître la mort d'Offerhaus, c'est en premier lieu la perte d'un homme doué de qualités humaines exceptionnelles. Offerhaus était un homme juste, un "honnête homme ". Il était un libéral dans le meilleur sens du terme: un homme attaché aux traditions, ayant le sentiment des formes et du décorum, mais en même temps sans préjugés, prêt à juger toute nouvelle tendance, toute idée originelle sur ses propres mérites et à se faire une opinion indépendante làdessus.

Sa figure impassible de régent cachait une nature sensible et délicate, la nature d'un homme plein d'un vif intérêt pour autrui. Cette nature se manifestait en tout premier lieu dans ses rapports avec les étudiants. Offerhaus n'était pas seulement professeur, il méritait vraiment ce titre d'honneur. Il était le coryphée de sa profession, des matières qu'il enseignait, mais malgré cela il ne manquait jamais d'apporter le plus grand soin possible à la preparation de ses cours. Ce qui lui importait avant tout c'était de transmettre ses connaissances, de faciliter à ceux qui ne sont pas encore des juristes accomplis l'accès à la matière si pénible à pénétrer. C'est pourquoi il recherchait autant que possible le contact de ses étudiants dont il voulait faire la connaissance personnelle. Il s'intéressait à leurs problèmes personnels, à leurs difficultés qui, si souvent, exercent une fâcheuse influence sur les études. Offerhaus n'était pas seulement le conseiller scientifique de ses étudiants, il était aussi leur mentor personnel et, à leur tour, ils le vénéraient comme leur maître.

Dans sa biographie $M$. Kisch aussi y fait allusion en disant: "C'est notamment sous cet aspect que, dans leurs cœurs, il a gagné une place toute spéciale; leurs sentiments ne s'arrêtent pas au respect de ses connaissances, de la clarté de ses exposés et de sa clémence lors des examens; ils comportent en plus une sincère gratitude pour tout l'intérêt qu'il leur porte dans leur bonheur et malheur."

L'influence inspiratrice qu'Offerhaus exerçait sur ses étudiants se manifeste dans le grand nombre d'entre eux qui, malgré sa difficulté, ont choisi le droit international privé comme matière a option et dans le nombre exceptionnel de thèses de haute qualité écrites sous sa direction et dont pas moins de cinq ont obtenu la mention "cum laude ". Autre témoignage du grand respect que ses étudiants portaient à Offerhaus est la fondation, lors de sa démission, par ses anciens étudiants de la "Studiekring Offerhaus " où sous la direc- 
tion du maître une conférence avait lieu deux fois par an qui fut publiée ensuite.

Pour ses amis aussi Offerhaus était l'homme qui était toujours prêt à faire siens leurs problèmes et leurs difficultés et il ne manquait jamais de leur donner un conseil réfléchi. Il était l'arbitre-né, qui possédait par excellence toutes les qualités requises pour cette fonction: une grande intelligence, une impartialité parfaite, une intégrité au-dessus de tout doute, du tact, l'esprit d'à-propos. Ce sont ces qualités qui faisaient de lui le président idéal de la Commission d'Etat pour la codification du droit international privé et de La Conférence de la Haye, organes dans lesquels j'ai eu le grand privilège de collaborer avec lui pendant $\mathrm{I} 7$ ans. C'était une expérience unique de voir comment Offerhaus, de main de maître, dirigeait des réunions parfois pénibles, savait réconcilier des opinions opposées, comment dans les milieux internationaux aussi il était le primus inter pares, joignant l'autorité à l'amabilité, le sens pratique à l'humour, la fermeté au tact et à la diplomatie. Personne ne contestera que c'est en grande partie grâce à l'activité et à la capacité d'Offerhaus que La Conférence de La Haye, après la Seconde Guerre Mondiale, est sortie de sa léthargie, que sous sa présidence 14 conventions multilatérales ont été établies dont júsqu'ici 6 sont entrées en vigueur, tandis que les autres ont au moins un considérable effet "de rayonnement", que La Haye est redevenue ce qu'elle était au début de ce siècle: le centre de l'unification du droit international privé.

Offerhaus n'était pas homme à se prévaloir de ses travaux. Au contraire, dans l'intimité il exprimait souvent un sentiment d'insatisfaction, parce que les tâches dont il était chargé l'avaient empêché de fournir d'importantes contributions au droit international privé. En effet, il n'a jamais eu l'occasion d'écrire un magnum opus. Toutefois, on sous-estimerait ses mérites purement scientifiques si on les mettait au-dessous de ses grands succès dans le domaine pratique et organisateur. Il est fort regrettable que tant sa thèse que beaucoup de ses articles aient été écrits en néerlandais, par suite de quoi ils ne sont pas ou à peine accessibles aux experts du droit international privé à l'étranger. Notamment sa thèse, ses observations sur l'autonomie de la volonté, parues dans le volume I de cette Revue, ainsi que sa Conférence, publiée en 1963 , sur le problème de l'adaptation dans le droit international privé auraient été internationalement connus s'ils avaient été écrits dans une langue universelle. Toutes ses publications se caractérisent par une parfaite connaissance de la matière, une grande précision, un jugement équilibré et une argumentation concise. A titre d'exemple, accessible aux non-Néerlandais également, je cite son "opinion dissidente" dans l'arrêt de la Cour Internationale de Justice portant sur l'affaire Elisabeth Boll (Recueil des Arrêts 1958) où Offerhaus faisait fonction de juge ad hoc.

Nombre de publications portent sur les activités de La Conférence 
de La Haye à laquelle Offerhaus a consacré une grande partie de sa vie. Ses observations sur les activités de La Haye ne contiennent pas seulement des commentaires faisant autorité sur les conventions établies, mais elles traitent souvent de problèmes fondamentaux de droit international privé soulevés lors de la discussion d'un sujet au cours d'une Session. On n'a qu'à lire son article sur la Septième Session de la Conférence dans Clunet I952, p. I67o e.s. ou son article intitulé «La Conférence de La Haye de Droit International Privé, Expériences et perspectives " dans Annuaire Suisse de Droit International, 1959.

Mais avant tout il faut bien se rendre compte que tant la présidence de la Commission d'Etat pour la codification du droit international privé que la part que prend le président à la préparation des différents sujets pour la Conférence et la direction de la Conférence elle-même exigent une activité scientifique de la plus haute qualité. Si un président n'est pas complètement au courant des sujets et de tous les problèmes y relatifs, si sa connaissance de la matière n'égale pas celle du meilleur expert, il faillit à sa tâche. Quand on étudie les Actes de La Conférence depuis I95 I on ne peut manquer de voir qu'Offerhaus a toujours rempli ces conditions.

Point de doute qu'au point de vue scientifique Offerhaus a été un digne successeur des grands savants qui ont occupé avant lui la chaire de droit international privé à l'Université d'Amsterdam, à savoir T. M. C. Asser, D. Josephus Jitta et I. Henri Hijmans, dont l'œuvre et la signification ont fait l'objet d'un excellent article de sa main dans la "Festgabe Max Gutzwiller, Jus et Lex".

Dans la personne d'Offerhaus un grand savant et un grand homme nous a quittés pour toujours. Mais tous ceux qui l'ont connu garderont à tout jamais le souvenir d'un homme comme on n'en rencontre que rarement dans la vie. 